\title{
P20: Gesundheitsökonomische Aspekte der Krankenpflege am Beispiel der zukünftigen Potenziale eines pflegerischen Versorgungsangebots von Demenzkranken
}

\author{
Thorsten Zehlicke • Hans Hartweg
}

Online publiziert: 23. Oktober 2013

(C) Springer-Verlag Wien 2013

Einleitung: Auch in der Pflege werden Fach- und Führungskräfte zukünftige Trends vor dem Hintergrund einer gesundheitsökonomischen Sicht beurteilen müssen. Dies gilt besonders für Erkrankungen die durch den demografischen Wandel zukünftig stark zunehmen werden. Da kostenträgerseitig nicht zu erwarten ist, dass zukünftig mehr Finanzmittel zur Verfügung stehen, wäre ein ökonomischer Einsatz der Ressourcen wünschenswert.

Methodik: Darstellung der stationären Versorgung von Demenzkranken in Deutschland und Einschätzung der Verbesserungspotenziale. Anwendung des Prozessmanagements auf die Pflege von Demenzkranken sowie Beurteilung des Pflegeteams durch ein fundiertes Human Ressource Management.

Ergebnisse: Mit der Anwendung einerseits von speziellen Pflegekonzepten (z. B. Integrative Validation nach Richard), aber auch Managementmethoden (z. B. Human Ressources Management, Prozessmanagement) ist es möglich, den Pfle- geprozess für Demenzkranke zu optimieren und Ressourcen einzusparen. Wichtig im Bereich des Human Ressource Management sind die Schaffung einer angenehmen Teamatmosphäre sowie kleine Pflegegruppen. Durch konsequentes Prozessmanagement können Blind- und Fehlleistungen minimiert werden.

Diskussion: Bedingt durch den demografischen Wandel und einen Trend zur Singularisierung der Haushalte wird zukünftig eine immer größere Nachfrage nach professioneller Pflege zu erwarten sein. Mit der Anwendung von speziellen Pflegekonzepten, aber auch gesundheits- und pflegewissenschaftlichem Knowhow, können sowohl die Qualität als auch der Outcome der Therapie von Patienten gesteigert werden. Es kann zusätzlich gezeigt werden, dass eine sinnvolle Verwendung der Finanzmittel - Grundsätzen der Gesundheitsökonomie folgend - nicht mit Kostendämpfung gleichzusetzen ist, sondern für Fach- und Führungskräfte der Pflege ein wichtiges Hilfsmittel sein kann.

T. Zehlicke $\cdot$ H. Hartweg

Hochschule Fresenius, University of Applied Sciences, Management im Gesundheitswesen und Gesundheitsökonomie, Idstein, Deutschland 